\title{
An Optimal Reconstruction of the Human Arterial Tree from Doppler Echotracking Measurements
}

\author{
Laurent Dumas \\ Université de Versailles \\ Laboratoire de Mathématiques \\ 78035 Versailles, France \\ laurent.dumas@uvsq.fr
}

\author{
Pierre Boutouyrie \\ Université Paris Descartes \\ INSERM U970 \\ A.P. Hôpitaux de Paris HEGP \\ pierre.boutouyrie@egp.aphp.fr
}

\begin{abstract}
Starting from non invasive experimental measurements by Doppler echotracking, the human arterial tree of a given patient is numerically reconstructed.The chosen approach consists in building a simplified fluid/structure interaction model for each artery and to find the parameters of the network by solving an inverse problem.

The first reconstruction results of the lower arterial tree of a healthy patient are given and show a very good agreement with the echotracking measurements. Such numerical reconstruction, that includes in particular the estimation of the stiffness of each artery, will help for an early diagnosis of cardiovascular diseases.
\end{abstract}

\section{Categories and Subject Descriptors}

J.3 [Computer Applications ]: Life and medical sciences

\section{General Terms}

Algorithms

\section{Keywords}

optimization, arteries, fluid-structure interaction.

\section{INTRODUCTION}

The 3D numerical simulations of blood flows in the human arterial tree (see Figure 1) have achieved a very high level of accuracy in the last few years. However, the computational cost of such simulations is very important. Moreover they need the evaluation of many parameters that are very difficult to estimate.

With some hypotheses on the geometry of the arteries (cylindrical symmetry) and on the flow (parabolic profile), it is possible to use a simplified 1D fluid and structure model, far less costly and that uses a reduced number of parameters. The latter, either numerical or physical, are optimally estimated for each patient by solving an inverse problem based

Permission to make digital or hard copies of all or part of this work for personal or classroom use is granted without fee provided that copies are not made or distributed for profit or commercial advantage and that copies bear this notice and the full citation on the first page. To copy otherwise, to republish, to post on servers or to redistribute to lists, requires prior specific permission and/or a fee.

GECCO'12 Companion, July 7-11, 2012, Philadelphia, PA, USA Copyright 2012 ACM 978-1-4503-1178-6/12/07 ...\$10.00. on experimental measurements of the mean velocity and distortion of the arteries at few points of the arterial tree. As the cost function is obtained through the resolution of a complex system of coupled partial differential equations, an evolutionary approach is used to solve the associated inverse problem.

In view of a medical diagnosis of cardiovascular diseases, it is worth mentioning that the blood flow pressure and the stiffness of each artery, two important figures that are difficult to measure, are also outputs of the numerical reconstruction.

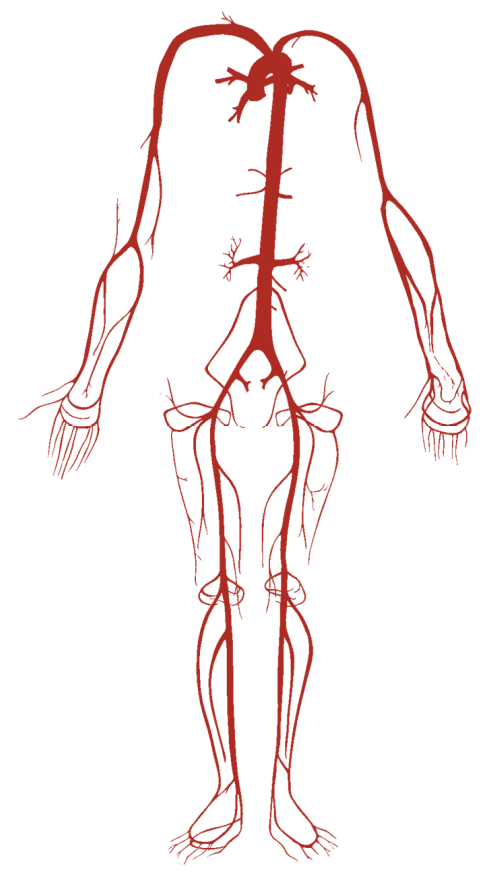

Figure 1: The human arterial tree

Section 2 presents the current cardiovascular risk estimation with the experimental device called echotracking. Section 3 is devoted to the description of the arterial tree model used here. Some examples of numerical simulations with this model are given in section 4 . Finally, an inverse problem resolution with evolutionary algorithms is presented in section 5 , leading to a more accurate estimation of the cardiovascular risk of a given patient. 


\section{THE CARDIOVASCULAR RISK}

\subsection{Echotracking measurements}

The echotracking device is a non invasive tool that can track the heart pulse wave with echo-doppler measurements at various points of the arterial tree (see [3]). It gives in particular the inter-adventicia diameter profile and the velocity profile at the artery center.

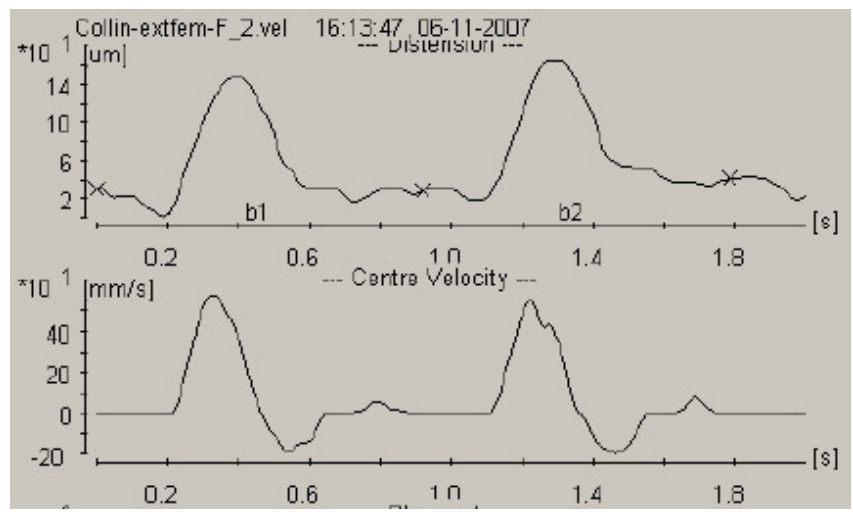

Figure 2: Example of echotracking measurements

The echotracking measurements used here have been done at the PARCC center of Hôpital Européen Georges Pompidou in Paris. An example of such data for the femoral artery is given on Figure 2.

\subsection{Estimation of the arterial stiffness}

The arterial stifness is a well-known index for predicting cardiovascular risks (see [4] for more details). It can be roughly estimated with echotracking measurements after computing the pulse wave velocity, also called PWV (see Figure 3).

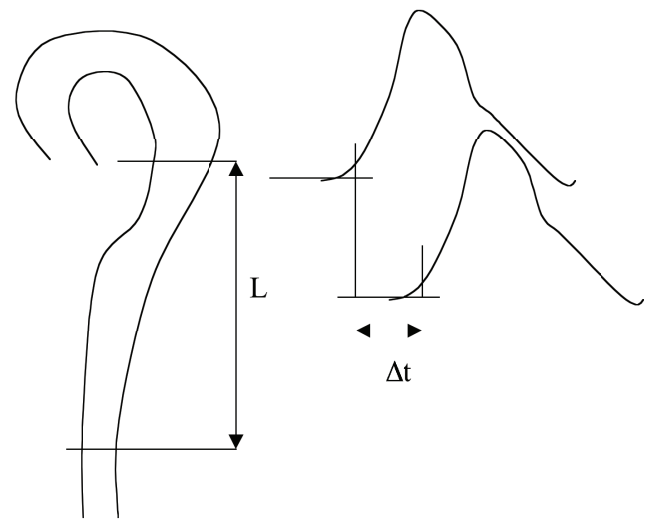

Figure 3: Pulse Wave Velocity estimation $\mathrm{PWV}=\mathrm{L} / \Delta \mathrm{T}$

The coefficient PWV is directly linked to the arterial stiffness with the Moens-Korteweg equation:

$$
P W V=\sqrt{\frac{E h}{2 r \rho}}
$$

where $E, h, r$ and $\rho$ are respectively equal to the vessel wall Young modulus, the wall thickness, the vessel radius and the blood density.

\section{THE ARTERIAL TREE MODEL}

We present here a new numerical method to estimate more accurately the arterial stiffness and other important parameters for a better cardiovascular risk prediction.

In the arterial tree, the blood flow velocity $u(t, x)$ and pressure $p(t, x)$ at time $t \in \mathbb{R}$ and position $x \in \Omega \subset \mathbb{R}^{3}$ satisfy the following 3D Navier Stokes equations:

$$
\left\{\begin{array}{l}
\frac{\partial u}{\partial t}+(u \cdot \nabla) u+\frac{1}{\rho} \nabla p+\operatorname{div}\left(\nu \frac{\nabla u+(\nabla u)^{T}}{2}\right)=0 \\
\operatorname{div}(u)=0
\end{array}\right.
$$

where $\rho$ and $\nu$ respectively denote the density and the kinematic viscosity of the blood. Because of the compliance of the arterial wall, theses equations must be coupled with an appropriate model of the vessel wall dynamics. Moreover, this compliance varies along the arterial tree and even along a single artery for instance because of the formation of atherosclerotic plaques or the presence of a stent.

In order to reduce both the computational time needed to solve these equations and the number of unknown parameters, a simplified 1D model has been developped by assuming that the artery has a cylindrical geometry with a circular cross section (see Figure 4).

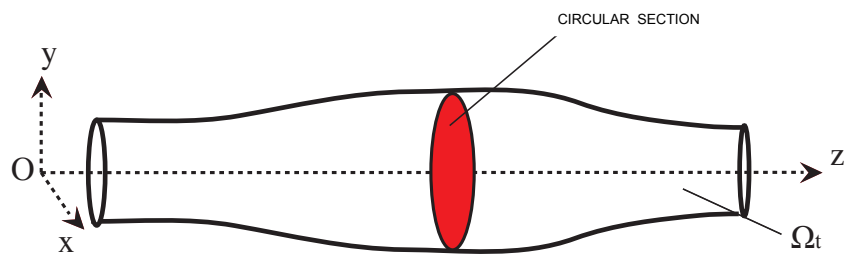

Figure 4: A simplified artery

After averaging the 3D Navier Stokes equations over each cross section, a system with two unknowns for each artery, the cross section area $A_{i}$ and the volumic flux $Q_{i}$, is derived (see [1] for more details):

$$
\left\{\begin{array}{l}
\frac{\partial A_{i}}{\partial t}+\frac{\partial Q_{i}}{\partial z}=0 \\
\frac{\partial Q_{i}}{\partial t}+\frac{\partial}{\partial z}\left(\frac{Q_{i}^{2}}{A_{i}}\right)+\frac{A_{i}}{\rho} \frac{\partial p}{\partial z}+K_{r} \frac{Q_{i}}{A_{i}}=0
\end{array}\right.
$$

where $A_{i}(t, z)$ is the cross section area at time $t$ and position $z \in\left[0, L_{i}\right]$ for the $i$-th artery, and $Q_{i}(t, z)$ the corresponding volumic flux. Note that in the more general case the total number of arteries in the human body is equal to 55 (see [2]).

In this system, $p_{i}(t, x)$ denotes the pressure in the $i$-th artery and is obtained through the following closure law:

$$
p_{i}(t, z)=\beta_{i}\left(\sqrt{A_{i}(t, z)}-\sqrt{A_{0, i}(z)}\right)
$$

where $A_{0, i}$ represents the section area of the $i$-th artery at rest .

The coefficients $K_{r}$ and $\left(\beta_{i}\right)_{1 \leq i \leq 55}$ appearing in the model are respectively linked to the blood viscosity and the arterial 
stiffness. A formal derivation from the $3 D$ to the $1 D$ model leads to the following expression for the parameter $\beta_{i}$, also called the rigidity coefficient of the $i$-th artery:

$$
\beta_{i}=\frac{4 \sqrt{\pi} h_{0, i} E_{i}}{3 A_{0, i}}
$$

where $h_{0, i}$ and $E_{i}$ respectively denote the thickness and the Young modulus of the $i$-th vessel wall.

The $1 \mathrm{D}$ model (2) can be rewritten in a conservative form, after omitting the index $i$ :

$$
\frac{\partial U}{\partial t}+\frac{\partial F(U)}{\partial z}=B(U)
$$

where $U=[A, Q]^{T}$ are called conservative variables, $F(U)$ and $B(U)$ are respectively the corresponding flux and source terms:

$$
F(U)=\left[\begin{array}{c}
Q \\
\frac{Q^{2}}{A}+\frac{\beta}{3 \rho} A^{\frac{3}{2}}
\end{array}\right], B(U)=\left[\begin{array}{c}
0 \\
-K_{r} \frac{Q}{A}
\end{array}\right]
$$

It can be observed that this system is hyperbolic, as the Jacobian

$$
H=\frac{\partial F}{\partial U}=\left[\begin{array}{cc}
0 & 1 \\
-\alpha \frac{Q^{2}}{A^{2}}+\frac{\beta}{2 \rho} A^{\frac{1}{2}} & 2 \frac{Q}{A}
\end{array}\right]
$$

has always two real eigenvalues, of opposite sign, for all the allowable values of $U, \beta$ and $\rho$ :

$$
\left\{\begin{array}{l}
\lambda_{+}=\frac{Q}{A}+\sqrt{\frac{\beta}{2 \rho}} A^{\frac{1}{4}}>0 \\
\lambda_{-}=\frac{Q}{A}-\sqrt{\frac{\beta}{2 \rho}} A^{\frac{1}{4}}<0
\end{array}\right.
$$

The associated characteristic variables have the following expressions:

$$
\left\{\begin{array}{l}
W_{+}=\frac{Q}{A}+2 \sqrt{\frac{2 \beta}{\rho}} A^{\frac{1}{4}} \\
W_{-}=\frac{Q}{A}-2 \sqrt{\frac{2 \beta}{\rho}} A^{\frac{1}{4}}
\end{array}\right.
$$

At each bifurcation, a system of six equations with six unknowns, namely the cross section and the flux for the corresponding three arteries, $\left(A_{1}, Q_{1}, A_{2}, Q_{2}, A_{3}, Q_{3}\right)$ on Figure 5 , is solved. It is made of:

(i) A continuity equation for the flux: $Q_{1}=Q_{2}+Q_{3}$.

(ii) Two total pressure conservation equations: $P_{T, 1}=P_{T, 2}$ and $P_{T, 1}=P_{T, 3}$ where $P_{T, i}=p_{i}+\frac{1}{2} \rho\left(\frac{Q_{i}}{A_{i}}\right)^{2}$.

(iii) Three compatibility conditions for the characteristic variables of the hyperbolic system, $\left(W_{+}\right)_{1},\left(W_{-}\right)_{2}$ and $\left(W_{-}\right)_{3}$.

The system is completed by the following initial conditions:

$$
\left\{\begin{array}{l}
A_{i}(0, z)=A_{0, i}(z) \\
Q_{i}(0, z)=0
\end{array}\right.
$$

and by two types of boundary conditions:

(i) At the entrance of the network: the value of the forward characteristic variable $W_{+}(t, 0)$ is imposed. It can be shown with relations (6) that it is equivalent to fix an entrance profile of the cross section area $A(t, 0)$. (ii) At each exit of the network: an equivalent resistance condition is imposed in order to simulate the downstream network (see Figure 5). The resistance rate is applied through a coefficient $R_{i} \in[0,1]$ at each exit of the network (see [2] for more details).

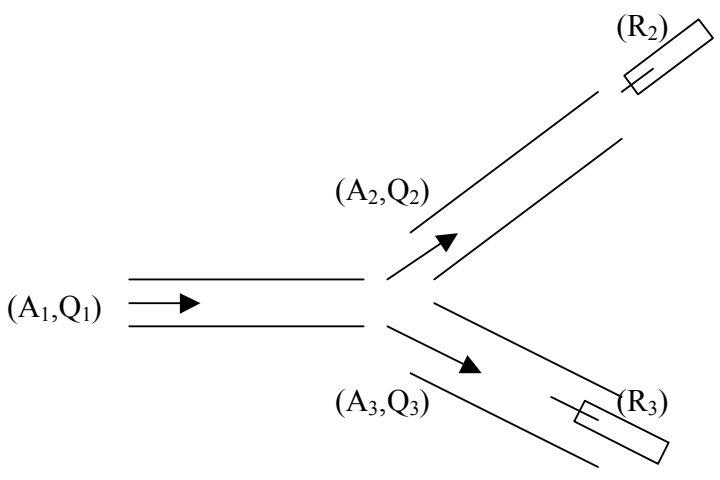

Figure 5: A simple network

Some preliminary works, made in particular by one of the present authors, have validated the accuracy of the 1D model compared to a full 3D fluid/structure model in the case of a straight artery (see $[5,6]$ ). In particular, it has been shown that the velocities and diameters of an artery computed with the $1 \mathrm{D}$ model were similar to the corresponding $3 \mathrm{D}$ values for a well chosen value of the rigidity coefficient $\beta$. Note that the optimal value of $\beta$ was not given by expression (4) but had to be fixed through an optimization process.

\section{THE DIRECT PROBLEM}

The equations of the 1D model are discretized in their conservative form (5) by using a second order Taylor Galerkin scheme.

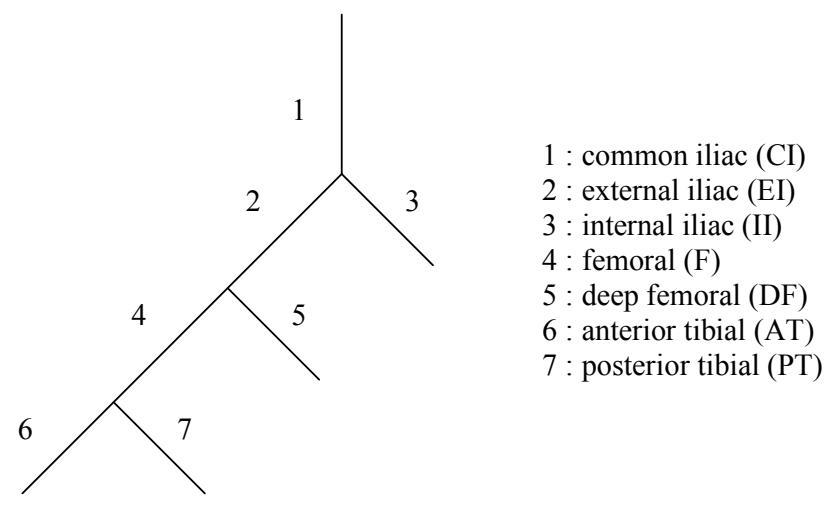

Figure 6: The lower arterial tree

Denote $\Delta t$ the chosen time step, then the vector of unknowns $U^{n}$ at time $t_{n}=n \Delta t$ satisfies the following timemarching scheme: 


$$
\begin{aligned}
U^{n+1}-U^{n}= & -\Delta t \frac{\partial}{\partial z}\left(F^{n}+\frac{\Delta t}{2}{\frac{\partial F^{n}}{\partial U}}^{n}\right) \\
& -\frac{\Delta t^{2}}{2}\left(\frac{\partial B^{n}}{\partial U} \frac{\partial F^{n}}{\partial z}-\frac{\partial}{\partial z}\left(\frac{\partial F^{n}}{\partial U} \frac{\partial F^{n}}{\partial z}\right)\right) \\
& +\Delta t\left(B^{n}+\frac{\Delta t}{2} \frac{\partial B^{n}}{\partial U} B^{n}\right)
\end{aligned}
$$

The spatial discretization is then done by using linear finite elements on the subdivision $\left(z_{i}\right)_{i \in\{0, \ldots, N\}}$ of $[0, L]$.

The human lower arterial tree is studied here as a first example of a numerical reconstruction (see Figure 6). It consists in a network made of seven arteries starting from the common iliac and ending at four arteries (internal iliac, deep femoral, anterior and posterior tibial).

The entrance profile, namely a cross section profile depicted on Figure 7), is issued from an echotracking measurement at the entrance of the common iliac artery of a healthy patient.

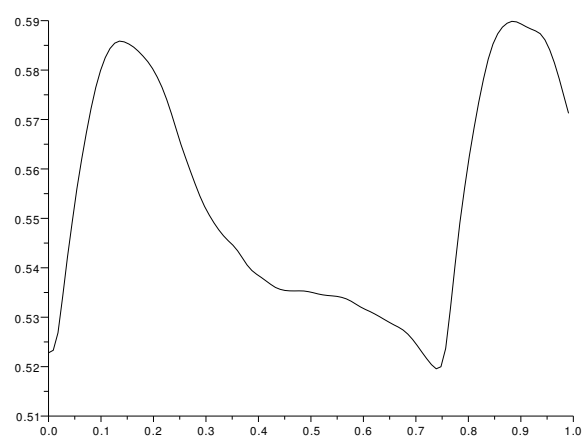

Figure 7: An entrance cross section profile

Three computations are presented here to show the influence of the physical parameters (the rigidity coefficients) and the numerical parameters (the exit resistances). The first computation uses the reference values that can be found in the literature (see [2] for instance) whereas in the second and third computations, these parameters have been slighty modified (see table 1).

\begin{tabular}{|l|c|c|c|}
\hline & Case 1 & Case 2 & Case 3 \\
\hline$\beta_{1}(\mathrm{CI})$ in $\mathrm{kg} / \mathrm{cm}^{2} / \mathrm{s}^{2}$ & 649 & 649 & 649 \\
\hline$\beta_{2}(\mathrm{EI})$ & 1493 & $\mathbf{1 9 0 0}$ & 1493 \\
\hline$\beta_{3}(\mathrm{II})$ & 3134 & 3134 & 3134 \\
\hline$\beta_{4}(\mathrm{~F})$ & 2559 & $\mathbf{3 1 0 0}$ & 2559 \\
\hline$\beta_{5}(\mathrm{DP})$ & 2652 & 2652 & 2652 \\
\hline$\beta_{6}(\mathrm{AT})$ & 5808 & $\mathbf{8 0 0 0}$ & 5808 \\
\hline$\beta_{7}(\mathrm{PT})$ & 9243 & 9243 & 9243 \\
\hline$R_{3}(\mathrm{II})$ adim. & 0.925 & 0.925 & $\mathbf{0 . 5}$ \\
\hline$R_{5}(\mathrm{DP})$ & 0.885 & 0.885 & $\mathbf{0 . 5}$ \\
\hline$R_{6}(\mathrm{AT})$ & 0.724 & 0.724 & $\mathbf{0 . 5}$ \\
\hline$R_{7}(\mathrm{PT})$ & 0.716 & 0.716 & $\mathbf{0 . 5}$ \\
\hline
\end{tabular}

Table 1: Three parameter sets

The results of the three computations are displayed on Figure 8 , here for the anterior tibial artery.
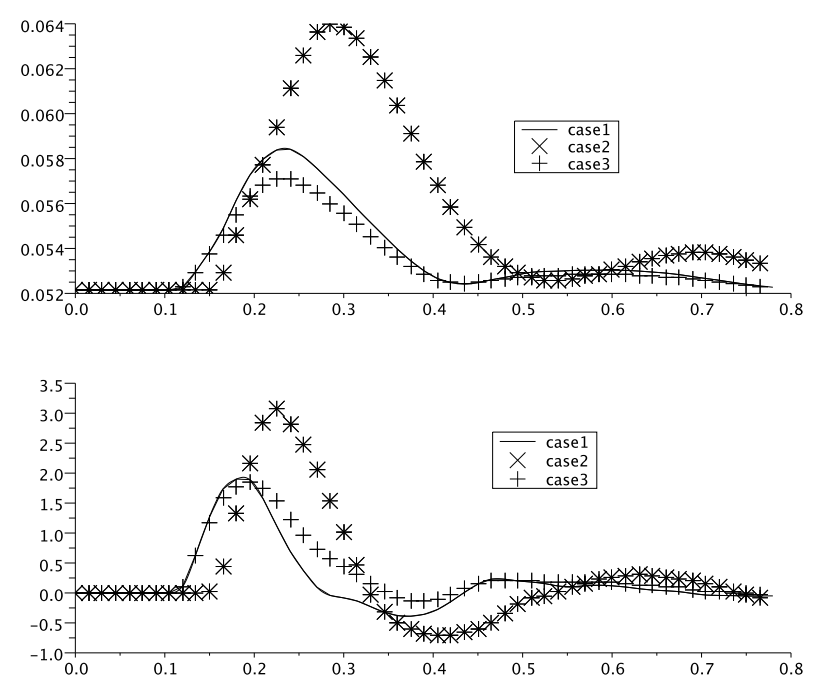

Figure 8: Cross section (top) and flux profiles (bottom) in the AT artery for three parameter sets.

They show that the numerical simulations are very sensitive to a minor change in few parameters of the simulation, respectively three rigidity coefficients $\beta_{i}$ and four exit resistances $R_{i}$. Knowing that the artery rigidity between two individuals can vary in a very large amplitude, it shows the importance to adapt the simulation to each patient.

The next section presents the method for building a patientspecific network that fits to its associated echotracking measurements.

\section{THE INVERSE PROBLEM}

\subsection{The cost function}

The resolution of the inverse problem is based on the minimization of an error type cost function between the numerical values and the corresponding experimental values. In the current case, only the experimental data on velocity profiles of four arteries have been used : the common iliac (index number $i=1)$, the external iliac $(i=2)$, the femoral $(i=4)$ and the anterior tibial artery $(i=6)$. All the velocities have been measured at the median position of each artery. Thus, the cost function writes as:

$$
J(\psi)=\sum_{k \in\{1,2,4,6\}} \frac{\left\|\left(\frac{Q_{k}}{A_{k}}\right)\left(t, \frac{L_{i}}{2}\right)-\left(V_{\text {echo }}\right)_{k}(t)\right\|_{L_{2}([0, T])}}{\left\|\left(V_{\text {echo }}\right)_{k}(t)\right\|_{L_{2}([0, T])}}
$$

The eleven parameters $\psi=\left(\beta_{1}, \ldots, \beta_{7}, R_{3}, R_{5}, R_{6}, R_{7}\right)$ on which the optimization process is performed are:

(i) Seven rigidity coefficients for the seven arteries : $\left(\beta_{i}\right)_{i \in\{1, \ldots, 7\}}$.

(ii) Four exit resistances: $\left(R_{i}\right)_{i \in\{3,5,6,7\}}$.

Note that other parameters are also included in the model, either geometrical $\left(L_{i}, A_{0, i}\right)$ or physical $(K r)$. As their influence is of lower order than the rigidity coefficients and the equivalent resistances, they have been kept fixed here. However, they can easily be included in the optimization process in the future. 


\subsection{The optimization method}

As seen before, the cost function $J$ is computed through a set of coupled fourteen PDE's and thus a gradient type method is difficult to implement here. For the optimization process, a genetic algorithm has been chosen as it is now classical for medical applications (see [7, 8, 9]).
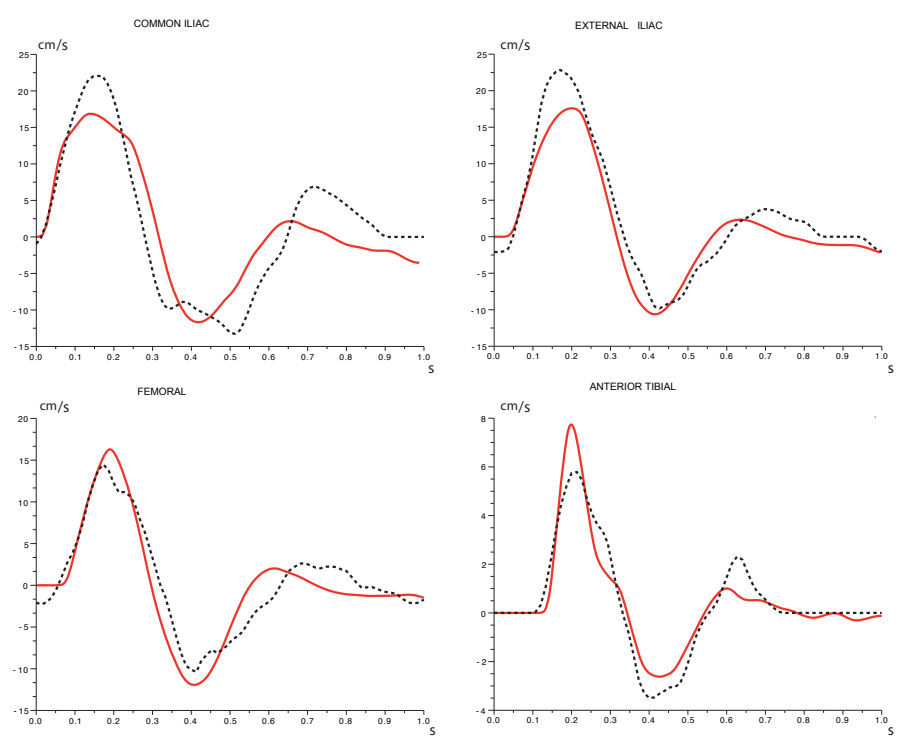

i...... EXPERIMENTAL VELOCITY

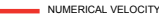

Figure 9: Numerical vs. experimental velocity profiles for the optimal network

In order to reduce the total computational time, an approximation $\tilde{J}$ of the exact cost function $J$ is constructed leading to the following metamodel-assisted GA:

- Generate initial population $P=\left\{\psi_{i} \in \mathcal{O}, 1 \leq i \leq N_{\text {pop }}\right\}$

- Until convergence do:

- Make approximate evaluations $\left\{\tilde{J}\left(\psi_{i}\right), 1 \leq i \leq N_{\text {pop }}\right\}$

- For the best $N_{\text {best }} \leq N_{\text {pop }}$ elements in terms of $\tilde{J}$, make exact evaluations of them.

- Make Darwinian operations (selection, crossover, mutation) on the population $P$ with respect to $\tilde{J}$.

- Generate the new population.

The number $\tau=\frac{N_{\text {best }}}{N_{\text {pop }}}$ representing the rate of exact evaluations at a given generation is equal to 1 for the first generations and is then linearly decreasing.

The metamodel method chosen here comes from the field of neural networks and is called RBF (Radial Basis Function) interpolation (see [10]). Suppose that the function $J$ is known on $N$ points $\left\{\psi_{i}, 1 \leq i \leq N\right\}$, the idea is to approximate $J$ at a new point $\psi$ by making a linear combination of radial functions of the type:

$$
\tilde{J}(\psi)=\sum_{i=1}^{n_{c}} \omega_{i} \Phi\left(\left\|\psi-\psi_{i}\right\|\right)
$$

where $\left\{\psi_{i}, 1 \leq i \leq n_{c}\right\} \subset\left\{\psi_{i}, 1 \leq i \leq N\right\}$ is the set of the $n_{c} \leq N$ nearest points to $\psi$ for the euclidian norm $\|$.$\| , on$ which an exact evaluation of $J$ is known. The function $\Phi$ is a radial basis function chosen here equal to

$$
\Phi(u)=\sqrt{u^{2}+r^{2}}
$$

(multiquadric RBF) for which the parameter $r>0$ is called the attenuation parameter.

The scalar coefficients $\omega=\left(\omega_{i}\right)_{1 \leq i \leq n_{c}}$ are then obtained by solving the linear least square problem of size $N \times n_{c}$ :

$$
\text { minimize } \operatorname{err}(\omega)=\sum_{i=1}^{N}\left(J\left(\psi_{i}\right)-\tilde{J}\left(\psi_{i}\right)\right)^{2}
$$

\subsection{Results}

The results are depicted on Figures 9 and 10. With the chosen approach, less than 200 exact evaluations of the cost function $J$ have been necessary to converge to the global minima of $J$.

On Figure 9, a comparison is done between the numerical and the experimental velocity profiles at the median position of the four artery CI, EI, F and AT for the optimal parameter set that has been obtained. It can be seen that the agreement between both profiles is excellent for every artery on a qualitative and also a quantitative viewpoint. Figure 10 compares the cross section area profiles for the same arteries. Even though these additional measurements have not been included in the computation of the cost function, here again, the agreement is also very good.
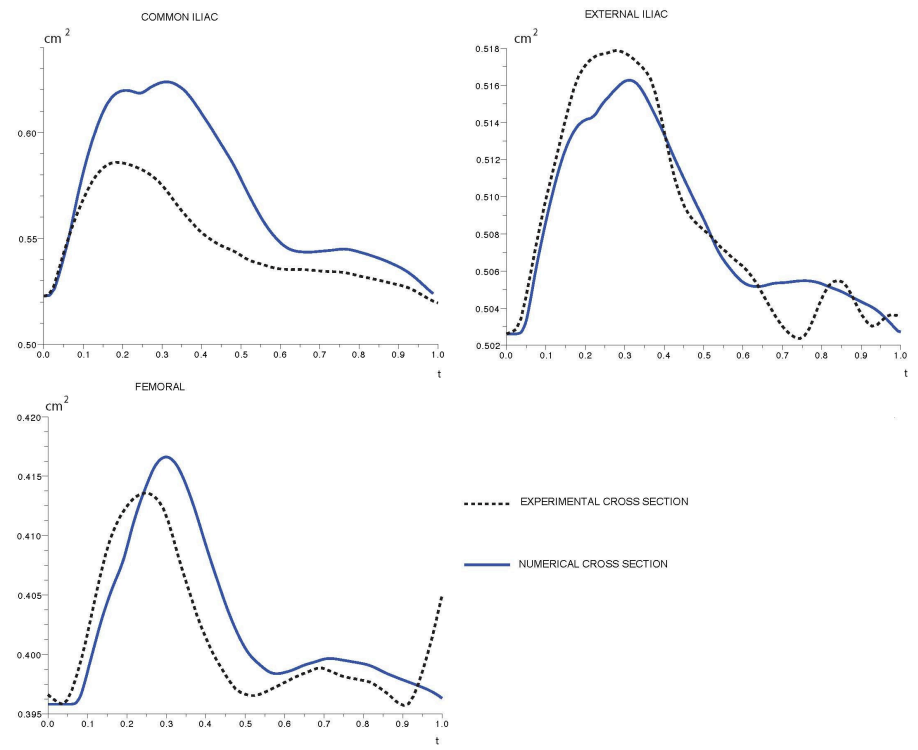

Figure 10: Numerical vs. experimental cross section profiles for the optimal network.

The same reconstruction process has been tested for other patients and has lead to the same quality of results, but with a different set of optimal parameters. It thus validates the whole reconstruction approach and also shows the importance of a patient specific method.

Finally, note also the computational time of a full reconstruction process, less than 24 hours, is compatible with a quick diagnosis of cardiovascular risks of a given patient and 
is far more precise than the one based on the PWV estimation presented in section 2 .

\section{CONCLUSION}

A new method for the reconstruction of the arterial tree and thus the prediction of cardiovascular risks of a given patient is presented here. It is based on non invasive Doppler echotracking measurements of blood flow velocity and arterial diameter profiles at few points of the arterial tree. The first results have shown that a perfect agreement between computation and experiment can be reached after an optimization process of the parameters of the numerical model. This kind of reconstruction process will help for a quick and early diagnosis of cardiovascular risks of a patient by giving accurate informations on the arterial stiffness and on the blood flow pressure through its whole arterial tree.

\section{REFERENCES}

[1] L. Formaggia, F. Nobile and A. Quarteroni, A One Dimensional Model for Blood Flow: Application to Vascular Prosthesis, Lecture Notes in Computational Science and Engineering, 19, p. 137-153, 2002.

[2] J. Alastruey, K.H. Parker, J. Peiro, S.J. Sherwin, Lumped parameter outflow models for 1-D blood flow simulations: Effect on pulse waves and parameter estimation, Communications in Computational Physics, 4(2), p. 317-336, 2008.

[3] H. Beaussier, O. Naggara, D. Calvet, R. Joannides, E. Guegan-Massardier, E. Gerardin, M. Iacob, B. Laloux, E. Bozec, J. Bellien, E. Touze, I. Masson, C. Thuillez, C. Oppenheim, P. Boutouyrie, S. Laurent, Mechanical and structural characteristics of carotid plaques by combined analysis with echotracking system and MR imaging, JACC Cardiovasc Imaging., 4(5), p. 468-477, 2011.
[4] F.Antonini-Canterin, S. Carerj, V. Di Bello, G. Di Salvo, S. La Carrubba, O. Vriz, D. Pavan, A. Balbarini, G.L. Nicolosi, Arterial stiffness and ventricular stiffness: a couple of diseases or a coupling disease? A review from the cardiologist's point of view, European Journal of echocardiography, Vol. 10, pp 36-43, 2009.

[5] V. Martin, F. Clément, A. Decoene and J.F. Gerbeau, Parameter identification for a one-dimensional blood flow model, Esaim Proc., 14, p. 174-200, 2005.

[6] L. Dumas, An optimal model of blood flow in arteries, proceedings of Engineering Optimization (ENGOPT), 2008.

[7] L. Dumas, L. El Alaoui, How Genetic Algorithms can improve a pacemaker efficiency, GECCO proceedings, p. 2681-2686, 2007.

[8] A. Blouza, L. Dumas, I. MBaye, Multi Objective Optimization of a Stent in a Fluid-Structure Context, GECCO proceedings, p. 2056-2060, 2008.

[9] Genetic and Evolutionary Computation, medical applications, Eds S. Smith, S. Cagnoni, John Wiley and Sons, 2011.

[10] L. Dumas, V. Herbert and F. Muyl, Comparison of global optimization methods for drag reduction in the automotive industry, Lecture Notes in Computer Science, 3483, p. 948-957, 2005. 